\title{
Self-pulsing in driven-dissipative photonic Bose-Hubbard dimers
}

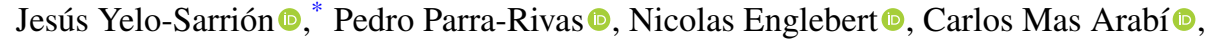 \\ François Leo ${ }^{\circ}$, and Simon-Pierre Gorza ${ }^{\circ}$ \\ OPERA-Photonique, Université libre de Bruxelles, 50 Avenue F. D. Roosevelt, CP 194/5, B-1050 Bruxelles, Belgium
}

(Received 1 April 2021; revised 14 July 2021; accepted 14 October 2021; published 22 November 2021)

\begin{abstract}
We report on the observation of nonlinear dynamics in driven-dissipative Bose-Hubbard dimers using a pair of coupled fiber ring resonators coherently driven by a single laser beam. We comprehensively explore the optical switching arising when scanning the detuning of the undriven cavity and demonstrate the key role played by the driven cavity detuning in the nonlinear regime. By driving the photonic dimer out of equilibrium, we observe the occurrence of robust self-switching oscillations.
\end{abstract}

DOI: 10.1103/PhysRevResearch.3.L042031

The spontaneous emergence of sustained periodic oscillations is a fascinating and ubiquitous phenomenon arising in nonlinear systems. It is associated with the breaking of translational symmetry in time and is encountered in various fields as diverse as chemistry, biology, mechanical engineering, or astrophysics to cite only a few $[1,2]$.

Since the seminal works of Lotka [3] and Volterra [4] a century ago in the context of chemistry and population dynamics in biology, respectively, it is now well known that such undamped oscillations may arise in nonlinear systems with coupled variables under continuous driving. An important example is the generation of infinite trains of pulses in the FitzHugh-Nagumo model of nerve membranes [5]. In optics, we can cite, for instance, self-pulsing in second-harmonic generation [6], in lasers between coupled longitudinal modes [7] or with continuous injected signal [8] or, more recently, between counterpropagating beams in a single Kerr ring resonator [9]. Rhythmogenesis refers to the emergence of oscillations from the coupling between two or more subsystems that show only steady states when uncoupled [10]. In this context, the driven-dissipative Bose-Hubbard (DDBH) model plays an essential role in physics, as it provides a canonical description of the dynamics between interacting bosons for open quantum systems [11]. In its simplest realization, only two macroscopic phase coherent wave functions are coupled to form a Bose-Hubbard dimer, also referred to as a bosonic Josephson junction. These junctions have been initially investigated with superconductors separated by a thin insulator [12] and with coupled reservoirs of superfluid helium [13]. Later, it was realized that they can be implemented with weakly coupled Bose-Einstein condensates in a macroscopic double-well potential [14] and in photonic systems with coupled semiconductor microcavities hosting polariton excitation [15]. However, besides Josephson effects,

\footnotetext{
*Corresponding author: jesus.yelo.sarrion@ulb.be

Published by the American Physical Society under the terms of the Creative Commons Attribution 4.0 International license. Further distribution of this work must maintain attribution to the author $(s)$ and the published article's title, journal citation, and DOI.
}

owing to their intrinsic nonlinearity, other striking phenomena such as anharmonic oscillations or macroscopic quantum selftrapping emerge in these latter systems [14-17]. Interestingly, under continuous excitation, it was theoretically predicted that sustained oscillations may take place in DDBH systems. This was shown not only for microcavity polaritons [17] but also for nonlinear optical cavities with two $[18,19]$ or more [20-22] coupled cavities. It is, however, only very recently that evidence of such self-pulsing was reported with polaritons, through its indirect spectral signature [23]. Moreover, coupled ring resonators host rich nonlinear dynamics and have recently attracted a lot of attention for frequency-comb generation in microresonators (see, e.g., Refs. [24-29]).

In this Research Letter, we report the observation of the spontaneous emergence of sustained oscillations induced by nonlinear self-interactions, between light beams propagating in two linearly coupled Kerr resonators. We consider asymmetrically excited photonic DDBH dimers [see Figs. 1(a) and 1(b)], in which the two cavity detunings $\left[\Delta_{L, R}\right.$; see also Eq. (1)] are distinct. We note that tuning these values is analogous to changing the single-particle energy of the two quantum states in bosonic Josephson junctions $[15,30]$. Here, the dimer is realized with passive fiber cavities. In contrast to other implementations of DDBH dimers, the detunings can easily be independently set or scanned without linear or nonlinear couplings between them. This is leveraged to shed light on the role played by the respective detunings in the emergence of self-pulsing.

The dynamics of the slowly varying envelope of the intracavity fields in coupled Kerr ring resonators can be described by a set of two coupled Lugiato-Lefever equations $[8,27]$. In single rings with normal dispersion, modulation instability occurs in a narrow range of parameters beyond the bistability threshold [31]. In addition, different works have recently shown that in coupled resonators, the local dispersion, induced by mode coupling, changes the instability spectrum and allows for the generation of stable localized patterns [24-26]. In our experiment, we carefully adjust the two cavity lengths to avoid such instability by ensuring that they both have the same free spectral range (FSR). The temporal walk-off as well as the group velocity dispersion are thus neglected in the model. Under these simplifications, the dynamics of the 

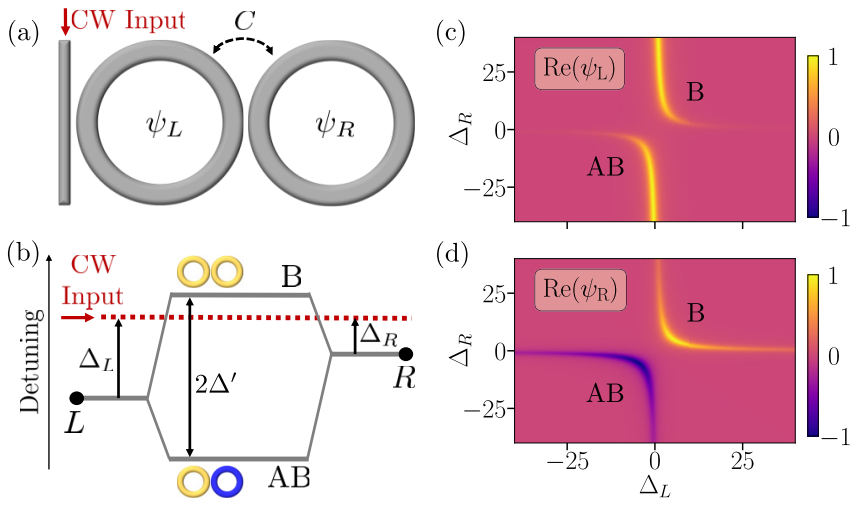

FIG. 1. (a) Schematic of an asymmetrically excited photonic dimer: The two resonators (L, R) are coupled (with normalized coupling strength $C$ ) and excited by an input beam coupled to the left resonator. (b) Detuning diagram, by analogy with the energy-level diagram of diatomic molecules. The coupling induces a resonance splitting giving rise to bondinglike $(\mathrm{B})$ and antibondinglike $(\mathrm{AB})$ modes. The resonance splitting is $2 \Delta^{\prime}=\sqrt{4 C^{2}+\left(\Delta_{L}-\Delta_{R}\right)^{2}}$, with $\Delta_{L, R}$ being the detunings from the driving laser. (c) and (d) Real part of the intracavity field amplitudes as a function of the detunings for $C=5$ [computed from Eq. (1) in the linear regime]. The fields are in phase (B mode) and out of phase (AB mode) for $\Delta_{L, R}>0$ and $\Delta_{L, R}<0$, respectively.

system is governed by the DDBH dimer model [24,32-34]. In dimensionless variables, it reads

$$
\begin{aligned}
\frac{d \psi_{L, R}}{d t}= & {\left[-1-i \Delta_{L, R}+i\left|\psi_{L, R}\right|^{2}\right] \psi_{L, R} } \\
& +i C \psi_{R, L}+S_{L, R},
\end{aligned}
$$

where the time $t=t^{\prime} \kappa / T_{R}$ with $t^{\prime}$ being the laboratory time, $T_{R}$ being the round-trip time, and $\kappa$ being the cavity loss coefficient (excluding the inter-resonator coupling). The detunings from the closest (single cavity) resonances are $\Delta_{j}=\delta_{j} / \kappa=$ $\left(m_{j} 2 \pi-\varphi_{j}\right) / \kappa(j=L, R)$, where $\varphi_{j}$ is the round-trip linear phase shift and $m_{j}$ is an integer number. $C=\sqrt{\theta_{12}} / \kappa$, with $\theta_{12}$ being the transmission coefficient of the coupler between the cavities. Finally, $\psi_{j}=A_{j} \sqrt{\gamma L / \kappa}, S_{L}=i \sqrt{P_{p} \gamma L \theta_{p} / \kappa^{3}}$, and $S_{R}=0$, where $A_{j}$ are the field amplitudes normalized such that the intracavity powers (expressed in watts) are given by $\left|A_{j}\right|^{2}=P_{j}, P_{p}$ is the driving power, $\theta_{p}$ is the transmission coefficient of the input coupler, $\gamma$ is the nonlinear parameter of the waveguide, and $L$ is the length of the resonators.

In the linear regime, the coupling splits the resonance, leading to an avoided crossing and the excitation of hybrid modes of the dimer, which is schematically shown in Fig. 1(b). In analogy to molecular physics, the resonance for positive (negative) detunings $\Delta_{L, R}$ corresponds to the excitation of the bondinglike (antibondinglike) modes of the dimer for which the fields in the coupled cavities are in phase (out of phase) as seen in Figs. 1(c) and 1(d) for $C=5$ [15]. In the nonlinear regime, the on-site self-interaction gives rise to an intensitydependent shift of the resonance and to dynamical instabilities through the nonlinear scattering towards the hybrid modes [17].

The experimental investigation of the nonlinear dynamics of DDBH dimers is performed with two fiber cavities coupled by a $95 / 5$ coupler. Each resonator is composed of about $L=250 \mathrm{~m}$ of optical fiber ( $1.14 \mu$ s round-trip time) with a net normal dispersion. The two cavity detunings can be independently stabilized by means of piezoelectric fiber stretchers. The left cavity is synchronously driven through a 90/10 coupler by flat-top 470-ps pulses generated from a narrow-linewidth distributed feedback laser. This prevents the buildup of the Brillouin scattering radiation and lowers the intracavity average power needed to reach the self-pulsing regime. The intracavity powers $\left(\propto\left|\psi_{L, R}\right|^{2}\right)$ are simultaneously measured on an oscilloscope at the cavity drop ports (see Supplemental Material for a schematic of the full setup [35]). The loss in each cavity has been experimentally estimated to be $2 \kappa \sim 40 \%$, leading to $C=1.08$.

Equation (1) has three independent parameters for a specific coupling $C$ (the two detunings $\Delta_{L, R}$ and the pump amplitude $S_{L}$ ) which determine a three-dimensional parameter space. To theoretically investigate the nonlinear regime, we fix, as in the experiment, the driven cavity detuning $\Delta_{L}$ and compute the different attractors of the system and their stability in the $\left(\Delta_{R}, S_{L}\right)$ parameter space. We note that despite their apparent complexity, the corresponding nonlinear resonances can easily be interpreted in this subspace. We carried out the bifurcation analysis of Eq. (1) following a numerical path-continuation approach using AUTO-07P [36].

First, we consider $\Delta_{L}=1.75$ to show how the bistability comes up in coupled systems. This value is indeed just above the bistability detuning threshold $(\sqrt{3})$ for single resonators [37]. The theoretical and experimental linear responses of the dimer are reported in Figs. 2(b) and 2(c). By increasing $S_{L}$, optical bistability is initially encountered in a narrow $\Delta_{R}$ detuning range between the saddle-node bifurcation lines $\mathrm{SN}_{1}$ and $\mathrm{SN}_{2}$ in the phase diagram shown in Fig. 2(a). The theoretical resonances at $S_{L}=1.5$ are plotted in Fig. 2(d) and compared with the experimental transmissions reported in Fig. 2(e). We observe a hysteresis cycle on the left side of the resonance in agreement with the fixed nodes of Eq. (1). Beyond the driving value at which $\mathrm{SN}_{1}$ and $\mathrm{SN}_{2}$ meet at the cusp bifurcation $c_{12}$, the system becomes monostable again, until the emergence of a new pair of saddle nodes $\mathrm{SN}_{3}$ and $\mathrm{SN}_{4}$ through a second cusp bifurcation $\left(c_{34}\right)$. Theoretical resonances corresponding to this regime are shown in Fig. 2(f) for $S_{L}=2.8$. The experimental scans confirm the shape of the nonlinear resonance and the existence of a bistable region for $\Delta_{R}>0$ [see Fig. 2(g)].

The bifurcation analysis of the DDBH dimer model predicts that continuous solutions unstable against periodic perturbations can be encountered for $\Delta_{L} \gtrsim 2.1$ and that the instability leads to the emergence of stable oscillations. Such a situation is depicted in Fig. 3(a) for $\Delta_{L}=3.1$, where the bistable regions as well as the self-pulsing area in the $\left(\Delta_{R}, S_{L}\right)$ plane of the parameter space are plotted. At $S_{L}=2$, the two saddle-node curves $\left(\mathrm{SN}_{2}, \mathrm{SN}_{2}^{*}\right)$ are crossed when scanning $\Delta_{R}$. We note that these two lines are actually connected in coupled ring resonators as they stretch between adjacent resonances. At this driving amplitude, the photonic dimer has two stable states for all $\Delta_{R}$ values, except in the vicinity of the avoided mode crossing [see Fig. 3(d)], in stark contrast with the behavior at $\Delta_{L}=1.75$. Moreover, the upper state is completely disconnected from the lower one. Consequently, 



FIG. 2. (a) Phase diagram in the $\left(\Delta_{R}, S_{L}\right)$ parameter space for $\Delta_{L}=1.75$. The saddle-node bifurcations $\mathrm{SN}_{j}$ are plotted as green lines, and $c_{i j}$ mark cusp bifurcations. The dimer is bistable in the shaded green areas. (b), (d), and (f) Bifurcation diagrams showing the normalized intracavity power, in the driven (blue) and undriven (red) resonators as a function of $\Delta_{R}$, for $S_{L}=0.2$ (b), 1.5 (d), and 2.8 (f) [see also dashed lines in (a)]. Solid (dashed) lines represent stable (unstable) solutions of Eq. (1). (c), (e), and (g) Corresponding experimental forward and backward scans. The arrows show the direction of the scans.

the system cannot spontaneously jump to the high-power state by changing the detuning of the undriven cavity. In order to observe the two states in the bistable region, we perturb $\Delta_{L}$ to allow the system to switch to the higher state. It then falls back to the lower one while scanning $\Delta_{R}$ in the forward or the backward direction. This is seen in Fig. 3(e), where only the down-switching transition is reported for both scanning directions. The two $\Delta_{R}$ values at which the switching takes place coincide well with the theoretical location of $\mathrm{SN}_{2}$ and $\mathrm{SN}_{2}^{*}$ in Fig. 3(d).

At a slightly higher driving strength, two new saddle-node lines $\mathrm{SN}_{1}$ and $\mathrm{SN}_{5}$ appear as a necking bifurcation [38] is crossed [see $n_{51}$ in the inset of Fig. 3(a)]. At this point, $\mathrm{SN}_{2}^{*}$ touches the lower state (not shown). It is beyond that power that self-pulsing occurs. The $\mathrm{SN}_{5}$ line, however, quickly disappears through a cusp bifurcation at $c_{25}$. Just above the power corresponding to $n_{51}, \mathrm{SN}_{1}$ and $\mathrm{SN}_{2}$ are located on either side of the newly formed connection between the lower and upper states. An example is given in Fig. 3(f) for $S_{L}=2.1$. We show in the experimental scans [see Fig. 3(g)] that this leads to a hysteresis cycle that stretches between adjacent resonances. The system jumps to the lower state at $\mathrm{SN}_{2}$ in the forward
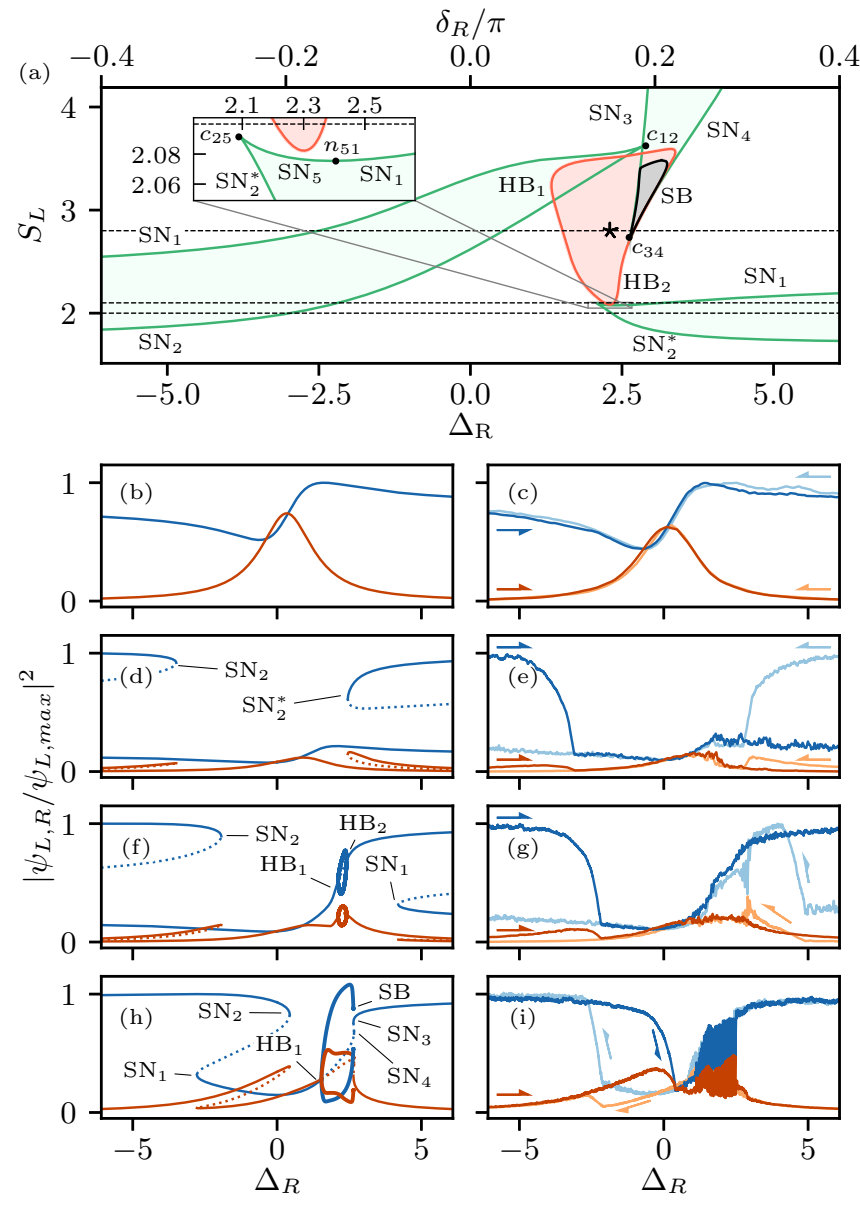

FIG. 3. Same as in Fig. 2, but for $\Delta_{L}=3.1$. (a) Phase diagram. The Hopf bifurcations $\mathrm{HB}_{1,2}$ (red lines) mark the boundaries of the self-pulsing region, and the Shilnikov bifurcation SB (black line) marks the boundaries of the area where there is no self-pulsing. $n_{41}$, necking bifurcation; $c_{i j}$, cusp bifurcation. The star indicates the parameters of the experiment reported in Fig. 4(a). (b), (d), (f), and (h) Normalized intracavity powers (thin lines) as a function of $\Delta_{R}$ at a driving $S_{L}$ value of $0.2,2.0,2.1$, and 2.8, respectively. The thick lines between the bifurcations $\mathrm{HB}_{1,2}$ show the maximum and minimum oscillation amplitudes. Note that in (h), $\mathrm{HB}_{2}$ (not shown for clarity) is located at $\mathrm{SN}_{4}$. (c), (e), (g), and (i) Corresponding forward and backward experimental scans. See main text for further explanation.

direction and then goes back to the upper state thanks to the connection in the avoided crossing region. In the backward direction, it first switches to the upper state at $\mathrm{SN}_{1}$; then it goes back down and stays on the lower state until the next resonance. Moreover, as seen in the experimental backward scan, the system spontaneously oscillates in a narrow range of $\Delta_{R}$ which coincides with the self-pulsing region located between the two Hopf bifurcations $\mathrm{HB}_{1}$ and $\mathrm{HB}_{2}$. Increasing the driving further, the self-pulsing region grows while $\mathrm{SN}_{1}$ moves away from the avoided crossing and appears on the other side of the resonance. The stable and unstable homogeneous solutions of Eq. (1) are plotted for $S_{L}=2.8$ in Fig. 3(h), as well as the boundaries of the oscillation amplitudes between the Hopf bifurcations. The measurements of the intracavity powers in the two resonators as $\Delta_{R}$ is scanned back and forth are reported in Fig. 3(i). They show a hysteresis cycle on the left 


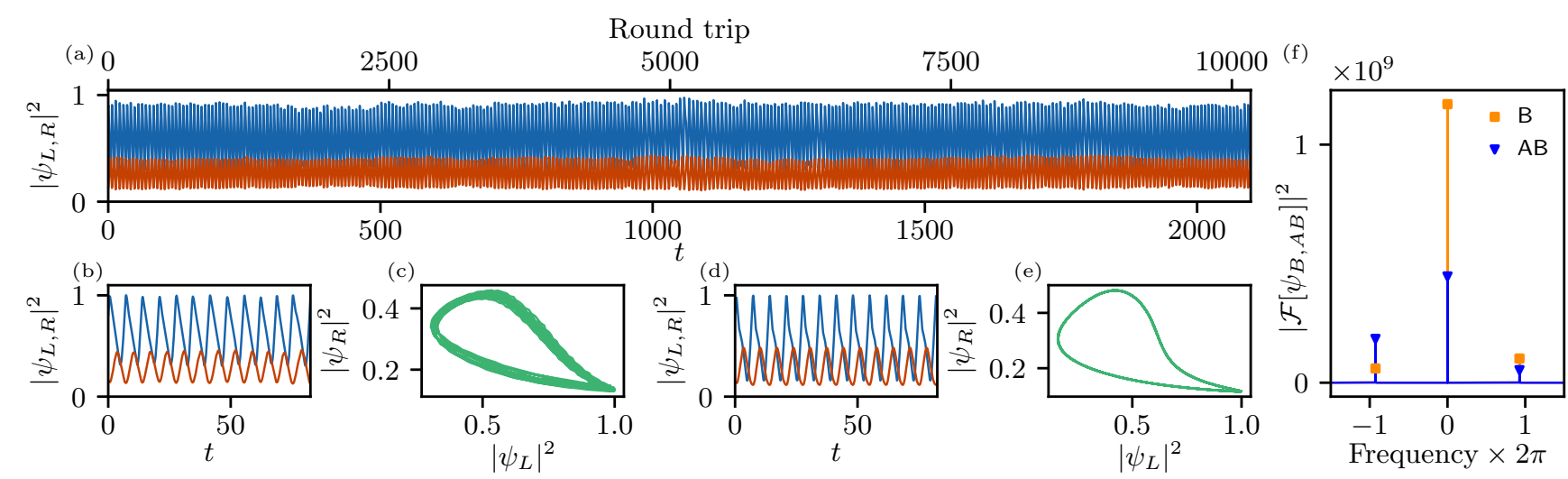

FIG. 4. (a) Normalized power in the left (blue) and right (red) resonators, recorded over more than 2000 lifetimes (10 000 round trips) and demonstrating periodic oscillations. The parameters are $S_{L}=2.8, \Delta_{L}=3.1$, and $\Delta_{R}=2.3$. (b) Zoom over $\approx 12$ periods and (c) corresponding trajectory in the $\left(\left|\psi_{L}\right|^{2},\left|\psi_{R}\right|^{2}\right)$-phase plane showing a limit cycle. (d) Sustained oscillations and (e) limit cycle obtained by numerical integration of Eq. (1). (f) Spectrum of the hybrid modes ( $\mathcal{F}$ denotes the discrete Fourier transform operator) corresponding to the self-pulsing shown in (d) and (e).

and self-starting oscillations on the right, whose locations are in excellent agreement with the bifurcation analysis of Eq. (1). We stress that the evolution of the nonlinear resonances with $\Delta_{R}$ for increasing $S_{L}$ and the emergence of self-pulsing for detunings $\Delta_{L}$ at which $\mathrm{SN}_{2}^{*}$ exists is not specific to lowcoupling DDBH dimers (see Supplemental Material for larger couplings [35]).

At a driving power close to $S_{L}=2.8$, the bifurcation diagram displayed in Fig. 3(a) shows that the system crosses a second cusp bifurcation $\left(c_{34}\right)$. This results in the emergence of two new continuous states separated by a pair of saddle-node bifurcations $\mathrm{SN}_{3,4}$. In between these lines, the self-pulsing undergoes a Shilnikov homoclinic bifurcation (SB) [33,39]. Here, the self-pulsing cycle is destroyed, and a homoclinic orbit appears, leading to type II excitability of the photonic dimer [40]. This is not reported because of the power limitation in our experiment. By further increasing $S_{L}$, only $\mathrm{SN}_{3}$ and $\mathrm{SN}_{4}$ remain, similar to the case shown in Figs. 2(f) and 2(g).

To prove that the oscillations seen in Fig. 3(i) correspond to self-pulsing, we stabilize the second cavity around $\Delta_{R}=2.3$, i.e., in the middle of the unstable region. A stable oscillation in both cavities is measured over 2000 cavity lifetimes, i.e., 10000 round trips [see Figs. 4(a) and 4(b)]. The trajectory in the $\left(\left|\psi_{L}\right|^{2},\left|\psi_{R}\right|^{2}\right)$-phase plane reported in Fig. 4(c) shows a limit cycle behavior consistent with the numerical simulation of Eq. (1) displayed in Fig. 4(e). This confirms the occurrence of self-pulsing in a driven-dissipative nonlinear bosonic Josephson junction. We note that the deviations from the average orbit in the experiment are attributed to the limitations of the stabilization technique of the two cavity detunings.

Self-pulsing in coupled cavities can be explained by the beating between the photons at the driving frequency and new photons generated by nonlinear scattering, with signal (idler) photons emitted at the frequency of the bondinglike (antibondinglike) mode $[19,23]$. The period of the self-pulsation is thus expected to be close to the beating period $2 \pi / \Delta^{\prime}=$ $2 \pi\left[C^{2}+\left(\Delta_{L}-\Delta_{R}\right)^{2} / 4\right]^{-1 / 2}$. However, this simple picture only holds in well-defined regions of the parameter space of DDBH dimers. Self-pulsing with a longer period is also possible and is typically found for $\Delta_{L} \approx 2 C$, for which the bondinglike mode is mainly excited (see Supplemental Material [35]). Besides, cascades of period doubling, an increasing oscillation period in the vicinity of a Shilnikov bifurcation, as well as chaotic pulsing, are also predicted to occur [33]. The spectrum of the self-pulsing plotted in Fig. 4(f) in the basis of the hybrid modes shows that in our experiment the bondinglike mode is the dominant mode at the driving wavelength and that the two main sidebands are composed of both hybrid modes. The measured oscillation angular frequency $(0.94)$ is also significantly smaller than the beating frequency $\Delta^{\prime}=1.16$.

In summary, we have explored, with ring cavities, the bifurcation structure of driven-dissipative Bose-Hubbard dimers. The ability to individually tune the two cavity detunings with respect to the driving field has been leveraged to study bifurcations in the $\left(\Delta_{R}, S_{L}\right)$ plane of the parameter space for different $\Delta_{L}$. We have shown the key role played by the driven cavity detuning $\left(\Delta_{L}\right)$ in the emergence of self-pulsing, which we have linked with the existence of the saddle-node $\mathrm{SN}_{2}^{*}$. The experiments are in very good agreement with the canonical driven-dissipative Bose-Hubbard dimer model, demonstrating that coupled ring resonators are well suited to study nonlinear Bose-Hubbard systems and, in particular, bosonic Josephson junctions. This model notably predicts the existence of a Shilnikov bifurcation, which enables excitability that may find applications in all-optical computing [41-43]. Our results are also relevant for the dynamics of hopping solitons, in photonic dimers and for solitons in a lattice of coupled resonators [28], which involve the synchronous oscillation of multiple modes.

The authors acknowledge fruitful discussions with Nicholas Cox and Emmanuel Gottlob. This work was supported by the Fonds de la Recherche Scientifique - FNRS under Grant No. PDR.T.0104.19 and the European Research Council (ERC) under the European Union's Horizon 2020 research and innovation program (Grant Agreement No. 757800). F.L., N.E., and P.P.-R. acknowledge the support of the Fonds de la Recherche Scientifique - FNRS. 
[1] G. Nicolis, Introduction to Nonlinear Science (Cambridge University Press, Cambridge, 1995).

[2] A. Jenkins, Self-oscillation, Phys. Rep. 525, 167 (2013).

[3] A. J. Lotka, Analytical note on certain rhythmic relations in organic systems, Proc. Natl. Acad. Sci. USA 6, 410 (1920).

[4] V. Volterra, Fluctuations in the abundance of a species considered mathematically, Nature (London) 118, 558 (1926).

[5] R. FitzHugh, Impulses and physiological states in theoretical models of nerve membrane, Biophys. J. 1, 445 (1961).

[6] P. D. Drummond, K. J. McNeil, and D. F. Walls, Nonequilibrium transitions in sub/second harmonic generation, Opt. Acta 27, 321 (1980).

[7] T. L. Paoli and J. E. Ripper, Coupled Longitudinal Mode Pulsing in Semiconductor Lasers, Phys. Rev. Lett. 22, 1085 (1969).

[8] L. A. Lugiato, L. M. Narducci, D. K. Bandy, and C. A. Pennise, Breathing, spiking and chaos in a laser with injected signal, Opt. Commun. 46, 64 (1983).

[9] M. T. M. Woodley, L. Hill, L. Del Bino, G.-L. Oppo, and P. Del'Haye, Self-Switching Kerr Oscillations of Counterpropagating Light in Microresonators, Phys. Rev. Lett. 126, 043901 (2021).

[10] I. R. Epstein and K. Showalter, Nonlinear chemical dynamics: Oscillations, patterns, and chaos, J. Phys. Chem. 100, 13132 (1996).

[11] C. Bruder, R. Fazio, and G. Schön, The Bose-Hubbard model: from Josephson junction arrays to optical lattices, Ann. Phys. (Berlin) 14, 566 (2005).

[12] K. K. Likharev, Superconducting weak links, Rev. Mod. Phys. 51, 101 (1979).

[13] S. V. Pereverzev, A. Loshak, S. Backhaus, J. C. Davis, and R. E. Packard, Quantum oscillations between two weakly coupled reservoirs of superfluid ${ }^{3} \mathrm{He}$, Nature (London) 388, 449 (1997).

[14] M. Albiez, R. Gati, J. Fölling, S. Hunsmann, M. Cristiani, and M. K. Oberthaler, Direct Observation of Tunneling and Nonlinear Self-Trapping in a Single Bosonic Josephson Junction, Phys. Rev. Lett. 95, 010402 (2005).

[15] M. Abbarchi, A. Amo, V. G. Sala, D. D. Solnyshkov, H. Flayac, L. Ferrier, I. Sagnes, E. Galopin, A. Lemaître, G. Malpuech, and J. Bloch, Macroscopic quantum self-trapping and Josephson oscillations of exciton polaritons, Nat. Phys. 9, 275 (2013).

[16] A. Smerzi, S. Fantoni, S. Giovanazzi, and S. R. Shenoy, Quantum Coherent Atomic Tunneling Between Two Trapped Bose-Einstein Condensates, Phys. Rev. Lett. 79, 4950 (1997).

[17] D. Sarchi, I. Carusotto, M. Wouters, and V. Savona, Coherent dynamics and parametric instabilities of microcavity polaritons in double-well systems, Phys. Rev. B 77, 125324 (2008).

[18] Y. Ekiolu and J. Petráček, Dynamical analysis of double-ring resonator with non-instantaneous Kerr response and effect of loss, Opt. Quantum Electron. 47, 3323 (2015).

[19] V. Grigoriev and F. Biancalana, Resonant self-pulsations in coupled nonlinear microcavities, Phys. Rev. A 83, 043816 (2011).

[20] B. Maes, M. Fiers, and P. Bienstman, Self-pulsing and chaos in short chains of coupled nonlinear microcavities, Phys. Rev. A 80, 033805 (2009).

[21] J. Petráček, Y. Ekiolu, and A. Sterkhova, Simulation of self-pulsing in Kerr-nonlinear coupled ring resonators, Opt. Commun. 318, 147 (2014).

[22] A. Armaroli, P. Feron, and Y. Dumeige, Stable integrated hyperparametric oscillator based on coupled optical microcavities, Opt. Lett. 40, 5622 (2015).
[23] N. Carlon Zambon, S. R. K. Rodriguez, A. Lemaitre, A. Harouri, L. Le Gratiet, I. Sagnes, P. St-Jean, S. Ravets, A. Amo, and J. Bloch, Parametric instability in coupled nonlinear microcavities, Phys. Rev. A 102, 023526 (2020).

[24] X. Xue, Y. Xuan, P.-H. Wang, Y. Liu, D. E. Leaird, M. Qi, and A. M. Weiner, Normal-dispersion microcombs enabled by controllable mode interactions, Laser Photonics Rev. 9, L23 (2015).

[25] S. A. Miller, Y. Okawachi, S. Ramelow, K. Luke, A. Dutt, A. Farsi, A. L. Gaeta, and M. Lipson, Tunable frequency combs based on dual microring resonators, Opt. Express 23, 21527 (2015).

[26] S. Fujii, Y. Okabe, R. Suzuki, T. Kato, A. Hori, Y. Honda, and T. Tanabe, Analysis of mode coupling assisted Kerr comb generation in normal dispersion system, IEEE Photonics J. 10, 4501511 (2018).

[27] X. Xue, X. Zheng, and B. Zhou, Super-efficient temporal solitons in mutually coupled optical cavities, Nat. Photonics 13, 616 (2019).

[28] A. Tikan, J. Riemensberger, K. Komagata, S. Hönl, M. Churaev, C. Skehan, H. Guo, R. N. Wang, J. Liu, P. Seidler, and T. J. Kippenberg, Emergent nonlinear phenomena in a driven dissipative photonic dimer, Nat. Phys. 17, 604 (2021).

[29] O. B. Helgason, F. R. Arteaga-Sierra, Z. Ye, K. Twayana, P. A. Andrekson, M. Karlsson, J. Schröder, and V. Torres-Company, Dissipative solitons in photonic molecules, Nat. Photonics 15, 305 (2021).

[30] S. R. K. Rodriguez, A. Amo, I. Sagnes, L. Le Gratiet, E. Galopin, A. Lemaître, and J. Bloch, Interaction-induced hopping phase in driven-dissipative coupled photonic microcavities, Nat. Commun. 7, 11887 (2016).

[31] M. Haelterman, S. Trillo, and S. Wabnitz, Dissipative modulation instability in a nonlinear dispersive ring cavity, Opt. Commun. 91, 401 (1992).

[32] W. Casteels and C. Ciuti, Quantum entanglement in the spatialsymmetry-breaking phase transition of a driven-dissipative Bose-Hubbard dimer, Phys. Rev. A 95, 013812 (2017).

[33] A. Giraldo, B. Krauskopf, N. G. R. Broderick, J. A. Levenson, and A. M. Yacomotti, The driven-dissipative Bose-Hubbard dimer: phase diagram and chaos, New J. Phys. 22, 043009 (2020).

[34] N. Jebali, L. Bodiou, J. Charrier, A. Armaroli, and Y. Dumeige, Combining FDTD and coupled-mode theory for self-pulsing modeling in coupled nonlinear microring resonators, J. Opt. Soc. Am. B 37, 2557 (2020).

[35] See Supplemental Material at http://link.aps.org/supplemental/ 10.1103/PhysRevResearch.3.L042031 for more details on the experimental setup and additional experimental and theoretical results.

[36] E. J. Doedel, A. R. Champneys, T. F. Fairgrieve, Y. A. Kuznetsov, B. Sandstede, and X. Wang, AUTO-07P: Software for continuation and bifurcation problems in ordinary differential equations, Department of Computer Science, Concordia University, Montreal (2007), https://github.com/auto-07p/auto07p.

[37] S. Coen, M. Haelterman, P. Emplit, L. Delage, L. M. Simohamed, and F. Reynaud, Experimental investigation of the dynamics of a stabilized nonlinear fiber ring resonator, J. Opt. Soc. Am. B 15, 2283 (1998). 
[38] J. Prat, I. Mercader, and E. Knobloch, The 1:2 mode interaction in Rayleigh-Bénard convection with and without Boussinesq symetry, Int. J. Bifurcation Chaos Appl. Sci. Eng. 12, 281 (2002).

[39] P. Glendinning, Stability, Instability and Chaos (Cambridge University Press, Cambridge, 1994).

[40] E. M. Izhikevich, Neural excitability, spiking and bursting, Int. J. Bifurcation Chaos Appl. Sci. Eng. 10, 1171 (2000).

[41] K. Vandoorne, P. Mechet, T. Van Vaerenbergh, M. Fiers, G. Morthier, D. Verstraeten, B. Schrauwen, J. Dambre, and
P. Bienstman, Experimental demonstration of reservoir computing on a silicon photonics chip, Nat. Commun. 5, 3541 (2014).

[42] Y. Shen, N. C. Harris, S. Skirlo, M. Prabhu, T. Baehr-Jones, M. Hochberg, X. Sun, S. Zhao, H. Larochelle, D. Englund, and M. Solačić, Deep learning with coherent nanophotonic circuits, Nat. Photonics 11, 441 (2017).

[43] J. Feldmann, N. Youngblood, C. D. Wright, H. Bhaskaran, and W. H. Pernice, All-optical spiking neurosynaptic networks with self-learning capabilities, Nature (London) 569, 208 (2019). 